\title{
GESTÃO ESTRATÉGICA DE RECURSOS HUMANOS: \\ quais práticas influenciam a avaliação dessa função pelos membros organizacionais?
}

\section{1- Camila Camargo}

Doutora em Administração pela Pontifícia Universidade Católica do Paraná (PUC-PR), Brasil.

Professora do Departamento de Administração Geral e Aplicada da Universidade Federal do Paraná (DAGA/UFPR), Brasil. caca.adm@gmail.com

http://lattes.cnpq.br/9804081697738477

\author{
Diego Maganhotto Coraiola - Editor Geral \\ Editor responsável pela submissão: \\ Diego Maganhotto Coraiola. \\ Artigo analisado via processo de revisão duplo cego (Double-blind). \\ Recebido em: 22/08/2014 \\ Aprovado em: 22/09/2014 \\ Última Alteração: 24/09/2014
}

* Contato Principal: Rua Espírito Santo, 411. Guaíra, Curitiba - PR, Brasil. CEP: 80630-200. 


\title{
GESTÃO ESTRATÉGICA DE RECURSOS HUMANOS: QUAIS PRÁTICAS INFLUENCIAM A AVALIAÇÃO DESSA FUNÇÃO PELOS MEMBROS ORGANIZACIONAIS?
}

\section{RESUMO}

Este artigo apresenta uma análise da influência das práticas de recursos humanos sobre a avaliação que os funcionários fazem da Competência da Gestão de Recursos Humanos nas suas organizações. Tal objetivo foi levado a termo com a realização de um levantamento com 347 funcionários de 8 organizações de serviços. Foram testadas nove hipóteses acerca da relação entre as práticas de RH e a avaliação da Competência da Gestão de RH dessas organizações. Por meio da aplicação de uma Análise de Regressão Múltipla verificou-se que a consideração de variáveis de recompensa (Remuneração e Benefícios) não altera substancialmente o poder explicativo do modelo acerca da variação na atitude dos funcionários em relação à Competência da Gestão de $\mathrm{RH}$, o que coloca maior peso sobre as variáveis denominadas Condições de Trabalho. Entre as Condições de Trabalho a variável Comunicação é a que apresenta maior influência sobre o julgamento da Competência da Gestão de RH. Tal achado coloca em primeiro plano o desenvolvimento das ações de Comunicação para reconhecimento do papel estratégico da Gestão de Recursos Humanos.

\section{Palavras-Chave}

Práticas de recursos humanos; gestão de recursos humanos; competência, status.

\section{STRATEGIC HUMAN RESOURCE MANAGEMENT: WHICH PRACTICES INFLUENCE ASSESSMENT OF THIS FUNCTION BY ORGANIZATIONAL MEMBER?}

\begin{abstract}
This paper provides an analysis of the influence of human resource practices on the evaluation that employees make related to Competence of Human Resource Management in their organizations. This purpose was brought to an end with the completion of a survey of 347 employees in eight service organizations in Brazil. We tested nine hypotheses about the relationship between HR practices and assessing the Competence of HR Management of these organizations. Through the application of a Multiple Regression Analysis it was found that the consideration of compensation variables (Compensation and Benefits) does not markedly modify the model's explanatory power about the change in the attitude of employees concerning the Competence of HR Management, which gives much importance on the variables called Working Conditions. Among Working Conditions the variable Communication is the one, which has more influence on the judgment of Competence of HR Management. This finding places at the forefront the development of social communication for recognizing the strategic role of Human Resource Management.
\end{abstract}

\section{Keywords}

Human resource practices; human resource management; competence; status. 


\section{Introdução}

A preocupação com a valorização da Gestão de Recursos Humanos e a justificativa de seu papel tem contribuído para geração de uma série de hipóteses entre as práticas que dão corpo aos sistemas de recursos humanos e seus consequentes (Lawson \& Hepp, 2001; Ramlall, 2003; Paauwe, 2009; Janssens \& Steyaert, 2009). Decorre daí, por exemplo, a investigação sobre a associação entre práticas de recursos humanos (HRM - Human Resource Management Practices) e o desempenho (Paauwe, 2009). A necessidade em posicionar a Gestão de RH como um conjunto de práticas essenciais às organizações tem levado pesquisadores e outros especialistas da área a reforçar seus aspectos estratégicos para acionistas e funcionários, sobretudo à alta gerência (Mamman \& Rees, 2004).

Nessa linha de raciocínio, a avaliação das práticas e iniciativas de RH tem ocorrido predominantemente a partir de critérios globais, tais como; eficiência, inovação e desempenho econômico (Ramlall, 2003; Boudreau \& Ramstad, 2006; Paauwe, 2009; Janssens \& Steyaert, 2009). Dessa perspectiva, pesquisadores têm se interessado em identificar em que medida as ações da Gestão de Recursos Humanos influenciam a variação de determinados resultados supraindividuais. Quando o indivíduo é considerado nesse processo as variáveis dependentes examinadas comumente são: satisfação; intenção de saída; comprometimento ou outras características atitudinais e comportamentais que atuam como mediadoras da influência das práticas de recursos humanos sobre o desempenho de grupo e da organização (e.g., Conway \& Monks, 2008; Zientara, 2009). Contudo, o modo como as práticas de recursos humanos são avaliadas no nível individual é uma questão ainda não explorada (Gvaramadze, 2008). Sabe-se pouco acerca de como os indivíduos no trabalho avaliam globalmente a Competência da Gestão de Recursos Humanos em suas próprias organizações.

Nesse ponto vale ressaltar que a avaliação da Gestão de Recursos Humanos, enquanto função, tem se dado quase que exclusivamente por meio da verificação da influência de suas práticas sobre variáveis que interessam, sobretudo, às organizações, e não à função RH como instituição social (Kochan, 2007). Tal limitação tem impossibilitado responder: O que a Gestão de Recursos Humanos precisa priorizar em suas ações para ser vista com bons olhos pelos funcionários? Tal questão endereça à avaliação da legitimidade dessa função nas organizações por seus membros, não obstante os julgamentos de acionistas e de outros stakeholders sejam igualmente importantes.

Logo, propõe-se no presente artigo delimitar quais são as práticas de recursos humanos que influenciam significativamente a avaliação que os funcionários fazem da gestão dessa área funcional. Desse modo, a principal contribuição do trabalho é promover a avaliação da gestão de RH, enquanto prática institucionalizada (Kochan, 2007), a partir da perspectiva do funcionário como ator social e não apenas como um recurso da organização, claramente descolado de um campo de maior espectro constituído por símbolos, crenças e valores. Esse exercício possibilita ir além da verificação da influência das práticas de Gestão de Recursos Humanos sobre fatores apenas organizacionais, como retorno sobre os ativos (Boudreau \& Ramstad, 2006). Assim, é possível delimitar quais práticas estão mais fortemente associadas à variação da atitude dos funcionários em relação à competência percebida dessa função em suas organizações.

Para consecução do objetivo supracitado o trabalho é apresentado em quatro tópicos após essa introdução: (i) quadro teórico de referência; (ii) procedimentos metodológicos; (iii) análise dos dados e discussão dos resultados; e, (iv) considerações finais.

\section{Quadro Teórico}

O quadro teórico de referência está dividido em dois subtópicos. O primeiro discorre sobre o status da Gestão de Recursos Humanos enquanto instituição social, o segundo trata do desenvolvimento das hipóteses sobre a avaliação das práticas dessa área funcional na perspectiva dos membros organizacionais.

\subsection{Status da Gestão de Recursos Humanos nas Organizações}

A fragilidade do status da Gestão de Recursos Humanos decorre de sua própria história. Pesquisadores afirmam desde a década de 1980 que a área tem se tornado cada vez mais eclética para responder às mudanças contextuais de ordem econômica, social e tecnológica (Mahoney \& Deckop, 1986). Essa diversidade interna da área também é apontada atualmente por Denisi, Wilson 
e Bitemam (2014) e Stone (2014) Wood, Tonelli e Cooke (2012) afirmam que essa diversidade tem figurado, inclusive, também como uma porta de entrada de diversos modismos gerenciais.

Outros autores acrescentam que grande parte das transformações e o alargamento das suas funções conduziram a uma crise de identidade e necessidade de reconhecimento e legitimidade, principalmente quando comparada a outras áreas organizacionais (Kochan, 2007, Kazmi \& Ahmad, 2001; Ferris, Hochwarter, Buckley, Harrell-Cook, \& Frink, 1999).

De acordo com Mahoney e Deckop (1986),o status atual da área está associado ao histórico da administração de pessoas e da função RH desde seu surgimento na literatura dos Estados Unidos e às mudanças que sucederam aquela época. Cabe destacar que, formalmente, a origem dessa área e da ênfase nas pessoas não está no experimento realizado em Hawthorne, o qual normalmente é atribuído como marco do início da Escola de Relações Humanas. De fato, o fenômeno da gestão de pessoas começou a ser definido mais claramente como objeto de análise da área de gestão a partir dos trabalhos de Mary Parker Follett (Graham, 1995) ainda na época de Frederick W. Taylor. Nesse sentido, como afirma Parker (1984) Mary Parker Follett foi contemporânea da Administração Científica apenas cronologicamente, pois intelectualmente ela já pertencia à era do homem social, destacando os papéis de grupos, conflitos e interações nas relações industriais.

Desde então, as relações de grupo no âmbito das organizações e o conjunto de atividades voltadas ao recrutamento, alocação e utilização de pessoas nas organizações tornaram-se elementos centrais de um campo intelectual com necessidade de atenção especializada em termos teóricos. A primeira disciplina aplicada ao estudo e prática da Gestão de Recursos Humanos foi a psicologia. Sob a denominação de psicologia industrial a gestão de RH foi submetida à análise em relação ao seu tratamento para questões como fadiga, curvas de aprendizagem, seleção e motivação no trabalho. Posterior à psicologia, a sociologia industrial dedicou-se, como bem ilustrado pelo experimento de Hawthorne, à investigação da interação social entre os membros da força de trabalho nas organizações e suas implicações. E quando a força dos trabalhadores se fez notar mais acentuadamente, por meio dos sindicatos em meio à depressão dos anos 1930, os estudos sobre a gestão de RH recorreram ao aporte de teorias econômicas para tratar do poder de barganha coletiva e do funcionamento do mercado de trabalho. Essas questões, somadas às mudanças do mercado de trabalho após a Segunda Guerra Mundial, acabaram por forçar a busca tanto por um melhor funcionamento do mercado de trabalho quanto da própria prática de Gestão de Recursos Humanos (Mahoney \& Deckop, 1986).

Dada a multiplicação de questões que se acumulavam sob a égide da gestão de RH, ou como denominada na época, Administração de Pessoas, o termo Relações Industriais (Industrial Relations) foi proposto como definição mais adequada para cobertura da análise do mercado de trabalho, das relações de trabalho e da administração de pessoas (Personnel Administration). Como resultado, a disciplina Relações Industriais destinou-se a articular a interdisciplinaridade entre psicologia, sociologia e economia para construção do conhecimento voltado à prática de gestão dos recursos humanos nas organizações. Contudo, como colocam Mahoney e Deckop (1986), apesar do crescimento dessa ênfase interdisciplinar no estudo das relações de trabalho, que foi dada pela Industrial Relations, a denominação Administração de Pessoas (Personnel Administration) se apresentou como um campo de investigação mais estreitamente definido nos anos 1950 e mais voltado para as práticas de negócios, bem como uma "tradução" mais funcional dessas práticas, tais como: recrutamento, seleção, treinamento e recompensa.

Mas só depois da articulação da Administração de Pessoas com a Teoria das Organizações ou Ciências Organizacionais (Organization Sciences), a qual tem ocorrido a partir dos anos 1960, é que a disciplina Gestão de Recursos Humanos (Human Resource Management) tem se voltado para a estruturação de um conhecimento mais coeso e integrado dentro de uma lógica de sistemas de RH. A partir daí houve ponderação de seus efeitos configuracionais sobre os resultados organizacionais (e.g., investigação de sistemas de compensação e sua influência sobre o retorno dos ativos organizacionais). Desde então, a Gestão de Recursos Humanos tem passado por uma mudança de ênfase; das relações humanas entre funcionários para o emprego das pessoas como recursos nas organizações, institucionalizando a partir daí a Human Resource Management no lugar da Personnel Administration.

Decorrente dessas mudanças, Kazmi e Ahmad (2001) reconheceram a presença de uma crise de identidade na Gestão de Recursos Humanos tanto no campo teórico quanto nas organizações. Mamman e Rees (2004) também afirmaram que, apesar do crescimento no interesse pela teoria e pela prática da área de Gestão de Recursos Humanos, muito trabalho ainda permanece por ser feito em comparação à necessidade de reconhecimento desse campo. Em um levantamento nos Estados Unidos com cento e dez gerentes de RH, Mamman e Rees (2004) identificaram que o papel do RH é 
desnecessariamente restrito, na perspectiva dos pesquisados, a questões de recompensa, participação nas decisões, treinamento e negociação. Nessa mesma linha, Kochan (2007) alerta para a necessidade de legitimidade, necessidade essa que se estende até a profissão gerência de recursos humanos (Lounsbury, Steel, Gibson, Drost, 2008).

Em conjunto, a crise de identidade dessa área funcional, e a necessidade de reconhecimento e legitimidade, contribuíram para que especialistas e pesquisadores buscassem ampliar a atuação da Gestão de Recursos Humanos. Na ânsia por maior reconhecimento, diversos pesquisadores têm sugerido modificações nas funções da Gestão de Recursos Humanos. Entre as mais urgentes está a natureza estratégica dessa função. Lawler III e Mohrman (2003) defendem a posição da Gestão de RH como um parceiro estratégico. Wright e McMahan (1992) também já haviam identificado duas décadas antes a eclosão de diferentes perspectivas teóricas no tratamento do conceito "Gestão Estratégica de Recursos Humanos". Na mesma linha de esforços de reconhecimento e legitimação da Gestão de Recursos Humanos, Clardy (2008) vê nessa área a responsabilidade principal pela construção das competências essenciais. Colbert e Kurucz (2007) atribuem a noção de sustentabilidade ao papel central do RH. Lawler III, Levenson e Boudreau (2004) reconhecem no uso de métricas e análises a possibilidade de justificação da importância estratégica do RH. Outros autores passaram a atribuir ao RH o papel de organização de consultoria interna (Vosburg, 2007), a responsabilidade pela ética nos negócios (Vickers, 2005), e até mesmo o gerenciamento de crises provocadas por desastres naturais (Premeaux \& Breaux, 2007).

No contexto brasileiro também podem ser apontadas várias transformações. Wood, Tonelli e Cooke (2012) apontam que o setor produtivo reconhece que a atual Gestão de Recursos Humanos não responde bem aos desafios, por exemplo, de atração e retenção de capital humano. Os autores chegam a afirmar que a literatura dessa área na mídia de negócios é triunfalista e associam as deficiências da área, como apontado também nos artigos internacionais, ao seu próprio histórico. $\mathrm{Na}$ visão desses autores a área de Gestão de Recursos Humanos no Brasil passou por duas fases principais: colonização (1950-1980) e neocolonização (1980-2010). A primeira fase, a de colonização, consistiu na implementação de práticas, programas e políticas trazidas de fora. Esse processo de importação se deu por meio das escolas de administração, mas também por meio das multinacionais que aqui se instalavam. Nesse sentido, o Brasil figurou nesse período como um solo fértil para lógicas e ferramentas de Gestão de Recursos Humanos que aqui chegavam. O segundo período, denominado pelos autores, de neocolonização, foi caracterizado por uma nova chegada de práticas e modelos de gestão de recursos humanos. A esse novo movimento de importação foram somadas uma tentativa de aproximação da área de Gestão de Recursos Humanos com os objetivos estratégicos das organizações e o desenvolvimento de um novo discurso que privilegiou o papel do líder em processos de mudança, os princípios de inovação e a valorização da competitividade e excelência nos negócios.

Essas tentativas de alargamento, modificação ou "evolução" (Mahoney \& Deckop, 1986) das funções, e por consequência, do conceito de Gestão de Recursos Humanos, contribuem, por diferentes vias, para o processo corrente de legitimação da função organizacional gestão de $\mathrm{RH}$ em meio a um momento de necessidade de tentativas de restauração da confiança na gestão das organizações, principalmente norte-americanas, por causa de uma série de escândalos corporativos (Kochan, 2007). Assim se justifica expor a necessidade de avaliação das organizações e suas práticas, enquanto instituições sociais, por diferentes audiências (e.g., acionistas, governos, clientes, fornecedores e funcionários). Logo, propõe-se nesse artigo verificar quais são as práticas de Gestão de Recursos Humanos que influenciam mais significativamente a avaliação que funcionários fazem da função RH em suas organizações. Como essa avaliação se dá a partir das atividades típicas dessa função, o próximo subtópico é destinado a apresentar os principais sistemas da Gestão de Recursos Humanos.

\subsection{As Práticas da Gestão de Recursos Humanos}

A Gestão de Recursos Humanos (Human Resource Management) é frequentemente apresentada em termos de seus principais sistemas constituintes de práticas (Collins \& Clark, 2003; Conway \& Monks, 2008). Vale destacar que o termo "práticas" é encontrado em uso na literatura dessa área sem as restrições de uma visão de fato praxeologicamente orientada em termos sociológicos. Isso significa que tal literatura se refere à noção de práticas de recursos como o simples agregado de atividades de um sistema ou função (Jamrog \& Overholt, 2004; Katou \& Budhwar, 2006) que é percebido pelos membros organizacionais. Sem os contornos construtivistas ou estruturacionistas da praxeologia, por exemplo, a ideia mais permissiva de práticas nessa 
disciplina possibilita verificar em que medida o julgamento das atividades dessa função (Innocenti; Pilati, \& Peluso, 2011; Rau, 2012; Thompson, 2011; Visser, 2010) acaba por influenciar a avaliação global que o funcionário faz do RH de sua organização. Essas práticas variam de modos de recompensa até treinamento e avaliação de desempenho. No presente estudo, para identificar as principais práticas ou sistemas de $\mathrm{RH}$ que poderiam influenciar mais significativamente a avaliação do $\mathrm{RH}$ nas organizações pesquisadas, foi realizado um levantamento entre os principais textos da literatura nacional e internacional sobre esse tópico, como aparece na Figura 1.

\begin{tabular}{|l|l|}
\hline Sistemas de Gestão de RH & Referência \\
\hline Variáveis: Condições de Trabalho & (FRANÇA, 2007; FISHER, 1998; \\
- Treinamento & RENCKLY, 2004; MATHIS; JACKSON, \\
- Descrição de cargos & $2008 ;$ BOHLANDER; SNELL; SHERMAN, \\
- Autonomia & $2005 ;$ DUTRA, 2002, FLEURY, 2002; \\
- Avaliação de Desempenho & BOXALL; PURCELL; WRIGHT, 2007; \\
- Ambiente Físico & KATOU; BUDHWAR, 2006; FERRIS; \\
- Comunicação & HOCHWARTER; BUCKLEY; HARRELL- \\
Variáveis: Recompensa & COOK; FRINK, 1999; RODRÍGUEZ; \\
- Remuneração & VENTURA, 2003; CAMELO; MARTIN; \\
- Benefícios & ROMERO; VALLE, 2004; COLLINS; \\
\hline
\end{tabular}

Figura 1 - Sistemas de Gestão de Recursos Humanos

Nota. Fonte: elaborado pela autora.

De acordo com o levantamento apresentado no Quadro 1, as práticas formais de Gestão de Recursos Humanos foram classificadas em dois fatores principais: (i) condições de trabalho; e, (ii) recompensa. Essa classificação em um ou outro fator obedeceu ao critério de similaridade entre nove sistemas identificados na literatura. Como Collins e Clarke (2003) defendem o isolamento de variáveis de incentivos ou recompensas das demais práticas de $\mathrm{RH}$, no exame da sua influência sobre outros consequentes, optou-se por seguir essa orientação também na presente pesquisa, sobretudo em função do debate acerca do maior ou menor peso dos sistemas de pagamento e outros incentivos sobre a atitude e comportamento do indivíduo no trabalho. Essa separação reforça a necessidade em se ponderar a influência da recompensa e das demais práticas ou sistemas de RH sobre a avaliação que os pesquisados fazem da Gestão de Recursos Humanos em suas organizações.

A partir da literatura foram então desenvolvidas as hipóteses do estudo. O primeiro fator, denominado Condições de Trabalho, agrega práticas de treinamento, descrição de cargos e tarefas, autonomia, avaliação de desempenho, oportunidades, ambiente físico, e comunicação. O segundo fator (Recompensa) contempla remuneração e benefícios. Vale notar que recrutamento é uma variável que não foi considerada porque, para os funcionários das organizações pesquisadas, que, portanto já estão ali empregados, seria mais adequado avaliar as oportunidades de crescimento. A variável dependente no modelo de análise é a avaliação da Competência da Gestão de Recursos Humanos nas organizações que fizeram parte do estudo. Essa variável foi escolhida por representar, em termos globais, a atitude do funcionário em relação a essa função organizacional. Como "competência", no senso comum, é sinônimo de boa execução e credibilidade optou-se pelo emprego do termo na presente investigação. Os enunciados das hipóteses acerca da relação entre as práticas de recursos humanos e a avaliação da Competência da Gestão de RH nas organizações pesquisadas são apresentados abaixo:

$H_{1}$ : Quanto mais positiva a avaliação dos treinamentos oferecidos ao funcionário maior será a sua avaliação em relação à Competência da Gestão de Recursos Humanos da organização.

$H_{2}$ : Quanto mais positiva a avaliação da descrição de cargos e tarefas para o funcionário maior será a sua avaliação em relação à Competência da Gestão de Recursos Humanos da organização.

$H_{3}$ : Quanto mais positiva a avaliação da autonomia no trabalho para o funcionário maior será a sua avaliação em relação à Competência da Gestão de Recursos Humanos da organização.

$H_{4}$ : Quanto mais positiva a avaliação do processo de acompanhamento de desempenho do funcionário maior será a sua avaliação em relação à Competência da Gestão de Recursos Humanos da organização. 
$H_{5}$ : Quanto mais positiva a avaliação das oportunidades de carreira para o funcionário maior será a sua avaliação em relação à Competência da Gestão de Recursos Humanos da organização.

$H_{6}$ : Quanto mais positiva a avaliação do ambiente físico de trabalho para o funcionário maior será a sua avaliação em relação à Competência da Gestão de Recursos Humanos da organização.

$H_{7}$ : Quanto mais positiva a avaliação da comunicação organizacional pelo funcionário maior será a sua avaliação em relação à Competência da Gestão de Recursos Humanos da organização.

$H_{g}$ : Quanto mais positiva a avaliação da remuneração para o funcionário maior será a sua avaliação em relação à Competência da Gestão de Recursos Humanos da organização.

$H_{g}$ : Quanto mais positiva a avaliação dos benefícios oferecidos ao funcionário maior será a sua avaliação em relação à Competência da Gestão de Recursos Humanos da organização.

\section{Procedimentos Metodológicos}

Os procedimentos metodológicos empregados no estudo são detalhados nas próximas subseções. O delineamento da pesquisa explicita o método de coleta de dados utilizado, a delimitação da população e o procedimento amostral. Posteriormente são apresentados os passos seguidos na construção das escalas de medida e as técnicas de tratamento dos dados que foram escolhidas para análise.

\subsection{Delineamento e Delimitação do Estudo}

Após a fase de análise da bibliografia sobre os sistemas de Gestão de Recursos Humanos, que auxiliou no desenvolvimento das hipóteses, foi construído um questionário e realizado um levantamento (survey) (Babbie, 1998, 2001) em oito organizações do setor de serviços de Curitiba (PR). Em relação à população do estudo fazem parte desse conjunto, todos os funcionários das empresas envolvidas no estudo. Optou-se pela amostragem por conveniência, considerando as empresas que permitiram mais fácil acesso aos pesquisadores (Neuman, 1997). Após a coleta de dados, a amostra final foi de trezentos e quarenta e sete $(n=347)$ respondentes.

\subsection{Escalas de Medida}

Para desenvolvimento dos indicadores do questionário estruturado (Neuman, 1997) foram observadas as definições constitutivas dos sistemas e práticas de Gestão de Recursos Humanos que constavam na literatura relacionada no Quadro 1. Procurou-se nessa etapa escrever um número mínimo de indicadores para cada sistema de recursos humanos, em função do número acentuado de variáveis independentes $(\mathrm{N}=9)$. Nessa mesma fase foram gerados os indicadores para avaliação da Competência da Gestão de Recursos Humanos (variável dependente). Após a escrita dos indicadores, quatro pesquisadores fizeram a validação de face do questionário. Foram sugeridas apenas algumas alterações para melhoria da compreensão pelos indivíduos que seriam pesquisados. Fez-se uso de uma escala intervalar (tipo Likert) de 10 pontos para avaliação dos indicadores. As opções variaram de (1) muito abaixo do esperado até (10) muito acima do esperado.

Desse modo, as práticas de recursos humanos e a Competência da Gestão de RH puderam ser comparadas com as expectativas de cada pesquisado. O número de indicadores variou de 2 a 4 por fator. O total de indicadores intervalares foi 29. Além das escalas intervalares foram coletados dados sobre (i) sexo; (ii) idade; (iii) cargo; e, (iv) tempo na empresa. As variáveis sexo e idade foram utilizadas para descrição da amostra final e as variáveis cargo e tempo na empresa foram empregadas como variáveis de controle no modelo de regressão múltipla. Cargo foi operacionalizado como variável dummy, por meio das seguintes categorias: (i) operacional, (ii) supervisão, coordenação ou cargo superior.

\subsection{Tratamento dos Dados}

Para o tratamento dos dados a base completa com as respostas dos entrevistados foi inicialmente submetida a uma análise exploratória (normalidade, linearidade, outliers, curtose e 
assimetria). Essa fase inicial teve por objetivo evidenciar a qualidade dos dados em termos de potencial para aplicação de técnicas estatísticas multivariadas. Na sequência, os dados intervalares foram submetidos a uma Análise Fatorial Confirmatória por meio da Modelagem de Equações Estruturais. Essa técnica foi escolhida em função da possibilidade de avaliação da validade convergente e discriminante das medidas. Após a verificação da qualidade das medidas foi observada a matriz de correlação das variáveis latentes. Essa matriz indicou correlações entre todos os indicadores. Assim, optou-se por empregar uma análise de regressão múltipla para o teste das hipóteses.

\section{Apresentação e Análise dos Dados}

Na caracterização da amostra a média de idade dos pesquisados foi 28 anos e 3,3 anos de tempo de empresa, com $52 \%$ de mulheres e $48 \%$ de homens. $78 \%$ dos entrevistados pertencem ao nível operacional, $12 \%$ ao nível de supervisão e $10 \%$ ao nível de coordenação ou superior. Na inspeção visual da qualidade dos dados não foi detectada não linearidade. Observou-se também que nenhuma das variáveis apresentou outliers com poder de interferência nos resultados, nem mais que $5 \%$ de missing values. No teste Kolmogorov-Smirnov todas as variáveis foram consideradas não normais. Entretanto, os índices de curtose estiveram entre 0,154 a -0,815 e de assimetria entre 0,479 a - - ,442, permitindo a aplicação da Análise Fatorial Confirmatória (AFC), que ocorreu por meio do software AMOS $^{\text {TM }}$ 18. Os resultados da AFC são detalhados nas tabelas 1 e 2.

\begin{tabular}{|c|c|c|c|}
\hline Indicadores & $\mathrm{CF}$ & AVE & $\mathrm{CC}$ \\
\hline \multicolumn{4}{|l|}{ Remuneração } \\
\hline Salário mensal pago pela empresa. & $1,000 *$ & \multirow[t]{3}{*}{0,77} & \multirow[t]{3}{*}{0,77} \\
\hline Compatibilidade do salário mensal com a média do mercado. & $0,983 \mathrm{t}$ & & \\
\hline Atualização salarial. & $0,933 \mathrm{t}$ & & \\
\hline \multicolumn{4}{|l|}{ Benefícios } \\
\hline Benefícios que vão além do tradicional salário mensal. & $0,985 \mathrm{z}$ & \multirow[t]{2}{*}{0,59} & \multirow[t]{2}{*}{0,74} \\
\hline Ajuda de custo para despesas pessoais (não relacionadas ao trabalho). & $1,000 *$ & & \\
\hline \multicolumn{4}{|l|}{ Comunicação } \\
\hline Capacidade de comunicação entre os vários departamentos na organização. & $0,973 \mathrm{t}$ & \multirow[t]{4}{*}{0,72} & \multirow[t]{4}{*}{0,95} \\
\hline Disseminação de informações conforme necessário. & $1,000 *$ & & \\
\hline Clareza das informações que são comunicadas. & $0,969 \mathrm{z}$ & & \\
\hline Rapidez na disseminação das informações mais importantes. & $0,989 \mathrm{t}$ & & \\
\hline \multicolumn{4}{|l|}{ Treinamento } \\
\hline Qualidade dos treinamentos na empresa. & $0,880 \mathrm{z}$ & \multirow[t]{3}{*}{0,84} & \multirow[t]{3}{*}{0,94} \\
\hline Quantidade dos treinamentos na empresa. & $1,000 *$ & & \\
\hline Frequência dos treinamentos na empresa. & $0,989 \mathrm{t}$ & & \\
\hline \multicolumn{4}{|l|}{ Autonomia } \\
\hline Autonomia no trabalho. & $0,940 \mathrm{z}$ & \multirow[t]{3}{*}{0,71} & \multirow[t]{3}{*}{0,88} \\
\hline Liberdade para fazer o trabalho sem monitoração e controle constante. & $1,000 *$ & & \\
\hline Liberdade de realizar o trabalho da forma mais adequada para o funcionário. & $0,741 \mathrm{t}$ & & \\
\hline \multicolumn{4}{|l|}{ Ambiente Físico } \\
\hline Ambiente de trabalho na empresa. & $0,861 \mathrm{t}$ & \multirow[t]{4}{*}{0,58} & \multirow[t]{4}{*}{0,92} \\
\hline Higiene no ambiente de trabalho. & $1,000 *$ & & \\
\hline Visual do local de trabalho. & $0,964 \mathrm{t}$ & & \\
\hline Temperatura no local de trabalho. & $0,846 \mathrm{t}$ & & \\
\hline \multicolumn{4}{|l|}{ Descrição de Cargos e Tarefas } \\
\hline Descrição das tarefas a serem realizadas na empresa. & 0,977 є & \multirow[t]{2}{*}{0,82} & \multirow[t]{2}{*}{0,90} \\
\hline Descrição das funções para cada cargo da empresa. & $1,000 *$ & & \\
\hline \multicolumn{4}{|l|}{ Avaliação de Desempenho } \\
\hline Avaliação do desempenho de funcionários que a empresa deve realizar. & $0,983 \mathrm{z}$ & \multirow[t]{3}{*}{0,80} & \multirow[t]{3}{*}{0,92} \\
\hline Acompanhamento do desempenho dos funcionários na empresa. & $0,994 \mathrm{t}$ & & \\
\hline Frequência das comunicações sobre o desempenho individual dos funcionários. & $1,000 *$ & & \\
\hline \multicolumn{4}{|l|}{ Oportunidade de Carreira } \\
\hline Oportunidades de carreira dentro da empresa. & $1,000 *$ & \multirow[t]{2}{*}{0,87} & \multirow[t]{2}{*}{0,93} \\
\hline Perspectiva de crescimento dentro da empresa. & $0,967 \mathrm{z}$ & & \\
\hline Avaliação da Gestão de RH & & & \\
\hline Forma de gestão do departamento de RH (recursos humanos) da empresa. & $1,000 *$ & 0,77 & 0,91 \\
\hline Capacidade de gestão do departamento de recursos humanos. & $0,972 \mathrm{t}$ & & \\
\hline Competência do departamento de recursos humanos na gestão de RH. & $0,889 \mathrm{t}$ & & \\
\hline
\end{tabular}

Tabela 1: Análise Fatorial Confirmatória (AFC) das Medidas

Fonte: dados primários.

Referências: CF-Carregamento; CC-Confiabilidade Composta $(0,70)$; AVE-Variância Média Extraída $(0,50)$.

‡ Carregamentos significativos a $p$-value $<0,01$.

*Significância não calculada em função de o parâmetro estar afixado em 1. 
Na execução da Análise Fatorial Confirmatória (AFC), empregou-se o método de estimação da Máxima Verossimilhança (Hair Jr., Anderson, Tatham, \& Black, 1995). Como supracitado, os dados não apresentaram distribuição normal, entretanto, Schumacker e Lomax (2004) defendem a aplicação de técnicas estatísticas multivaridas que pressuponham normalidade se os índices de curtose e assimetria não se apresentarem fora da amplitude de $\pm 1,00$. Na sequência foram analisadas as medidas de ajustamento do modelo, bem como validade e confiabilidade das variáveis latentes (fatores).

\subsection{Medidas de Ajustamento}

As medidas de ajustamento do modelo, conforme tabela 2, foram consideradas adequadas, estando próximas ou acima dos índices de referência. Os índices considerados foram: (1) ajuste absoluto (GFI, RMSEA); (2) ajuste incremental (AGFI, NFI e TLI); (3) ajuste parcimonioso (RFI, IFI e CFI) e; (4) ajuste geral (razão entre Qui Quadrado e Graus de Liberdade).

\begin{tabular}{lcl}
\hline Medidas de Ajustamento - Análise & Fatorial Confirmatória & Referência* \\
\hline Goodness of Fit Index (GFI) & 0,871 & 0,900 \\
Adjusted Goodness of Fit Index (AGFI) & 0,831 & 0,900 \\
Normed Fit Index (NFI) & 0,919 & 0,900 \\
Tucker-Lewis Coefficient (TLI) & 0,943 & 0,900 \\
Relative Fit Index (RFI) & 0,901 & 0,900 \\
Incremental Fit Index (IFI) & 0,953 & 0,900 \\
Comparative Fit Index (CFI) & 0,953 & 0,900 \\
Root Mean Square Error of Approximation & 0,060 & 0,080 \\
(RMSEA) & & 1,0 a 3,0 \\
Chi-Square/Degree of Freedom $\left(\mathrm{C}^{2}\right) /(d f)$ & 2,237 &
\end{tabular}

Tabela 2: Medidas de Ajustamento

Fonte: dados primários.

*Baseado em Hair Jr.et al. (1995) e Schumacker e Lomax (2004).

O GFI (índice de qualidade de ajuste) de 0,871 indica boa qualidade do modelo. O RMSEA (raiz do erro quadrático médio de aproximação) é de 0,060 , abaixo do valor máximo $(0,08)$. Quanto às medidas de ajuste incremental o AGFI (índice ajustado de qualidade de ajuste) é de 0,831 (próximo da referência 0,900) e o RFI $=0,901$ (Hair Jr.et al., 1995). Os indicadores NFI (índice de ajuste normado) $=0,919$, TLI (índice de ajuste não normado) $=0,943$, IFI (índice de ajuste incremental) = 0,953 e CFI (índice de ajuste comparativo) =0,953 também indicam bom ajuste. O valor $\mathrm{c}^{2} /$ Graus de liberdade é inferior a 3, como esperado.

\subsection{Validade e Confiabilidade das Medidas}

Confirmado o ajustamento do modelo por meio da AFC foram avaliadas validade e confiabilidade das medidas. A validade convergente das medidas foi atestada pela significância estatística $(p$-value $<0,05)$ da carga padronizada dos indicadores das variáveis latentes. Assegurouse também a validade discriminante comparando a correlação dos fatores (elevada ao quadrado) com a Variância Média Extraída (AVE) de cada variável latente (Fornell \& Larcker, 1981). A correlação ao quadrado não foi superior à AVE em nenhum caso e desse modo a validade discriminante das medidas foi comprovada. Para atestar a confiabilidade das medidas foram observadas a AVE e a Confiabilidade Composta (CC) de cada variável latente, calculadas a partir do carregamento padronizado dos indicadores apresentados na tabela 1. Todos os fatores (variáveis latentes) apresentaram confiabilidade composta (CC na tabela 1) acima de 0,70 e AVE acima de 0,50 , atestando assim suficiente consistência.

\subsection{Estatística Descritiva e Correlações entre as Variáveis Latentes}

Todas as variáveis latentes tiveram sua validade e confiabilidade assegurada por meio dos procedimentos apresentados acima com apoio da Análise Fatorial Confirmatória via Modelagem de Equações Estruturais. Esses procedimentos permitiram avaliar também a matriz de correlação dessas variáveis para verificar se existiam associações estatisticamente significativas que justificassem o emprego da Análise de Regressão Múltipla para o teste das hipóteses. Como 
apresentado na tabela 3 todas as variáveis latentes estão correlacionadas entre si, considerando um $p$-value $<0,01$. A mesma tabela também apresenta média e desvio padrão das variáveis latentes.

\begin{tabular}{llllllllllll}
\hline & $\bar{x}$ & $s$ & 1 & 2 & 3 & 4 & 5 & 6 & 7 & 8 & 9 \\
\hline 1. Descrição de Cargos & 6,06 & 1,81 & & & & & & & & & \\
2. Treinamento & 5,05 & 2,22 & 0,50 & & & & & & & & \\
3. Ambiente Físico & 6,53 & 1,90 & 0,61 & 0,43 & & & & & & & \\
4. Autonomia & 5,95 & 1,95 & 0,53 & 0,40 & 0,57 & & & & & & \\
5. Comunicação & 5,58 & 1,84 & 0,62 & 0,53 & 0,58 & 0,47 & & & & & \\
6. Av. Desempenho & 5,49 & 2,03 & 0,67 & 0,60 & 0,57 & 0,45 & 0,66 & & & & \\
7. Op. Carreira & 5,27 & 2,40 & 0,45 & 0,56 & 0,46 & 0,41 & 0,54 & 0,54 & & & \\
8. Remuneração & 4,79 & 1,82 & 0,30 & 0,37 & 0,32 & 0,32 & 0,36 & 0,34 & 0,43 & & \\
9. Benefícios & 4,04 & 2,02 & 0,36 & 0,51 & 0,35 & 0,32 & 0,52 & 0,49 & 0,48 & 0,53 & \\
10. Avaliação RH & 5,42 & 1,88 & 0,56 & 0,58 & 0,52 & 0,44 & 0,62 & 0,61 & 0,49 & 0,45 & 0,50 \\
\hline
\end{tabular}

\section{Tabela 3: Estatísticas Descritivas e Correlações}

Fonte: dados primários.

Todas as correlações são significativas $(p<0,01)$.

\subsection{Análise de Regressão Múltipla}

Para o teste das hipóteses optou-se pela análise de regressão múltipla. Essa análise permitiu dimensionar a influência do julgamento das práticas de RH sobre a avaliação da Competência da Gestão de Recursos Humanos das organizações que fizeram parte do estudo, de acordo com a opinião dos funcionários pesquisados. Nesses termos, a Competência da Gestão de Recursos

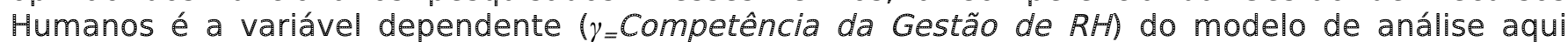
proposto e as práticas de $\mathrm{RH}$ as variáveis independentes ( $X_{1=}$ treinamento; $X_{2=}$ descrição de cargos; $X_{3=}$ autonomia; $X_{4=}$ avaliação de desempenho; $X_{5=}$ oportunidades; $X_{6=}$ ambiente físico; $X_{7=}$ comunicação; $X_{8=}$ remuneração; $X_{9=}$ benefícios $)$.

Ainda conforme a recomendação de Collins e Clarke (2003) foi testada separadamente a influência das variáveis de condições de trabalho das variáveis de recompensa. O primeiro modelo avaliou isoladamente a influência das variáveis de controle (Cargo e Tempo na Empresa). O segundo modelo teve como variáveis independentes as variáveis de controle e as variáveis de condições de trabalho, e o terceiro modelo contemplou, além das variáveis anteriores, também remuneração e benefícios. As equações de regressão dos modelos para teste das hipóteses (portanto, desconsiderando o modelo de teste das variáveis de controle) constam a seguir:

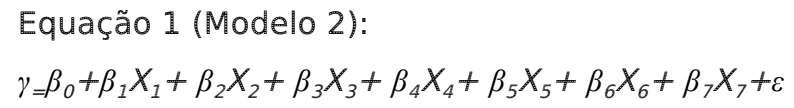

Equação 2 (Modelo 3):

$\gamma_{=} \beta_{0}+\beta_{1} X_{1}+\beta_{2} X_{2}+\beta_{3} X_{3}+\beta_{4} X_{4}+\beta_{5} X_{5}+\beta_{6} X_{6}+\beta_{7} X_{7}+\beta_{8} X_{8}+\beta_{9} X_{9}+\varepsilon$

Na tabela 4 são apresentados os resultados da análise de regressão múltipla hierárquica (com entrada em blocos de variáveis) para teste da influência das variáveis de controle sobre a Competência da Gestão de RH (Modelo 1), teste da influência das variáveis de Condições de Trabalho sobre a Competência da Gestão de RH (Modelo 2), e teste da influência das variáveis de Condições de Trabalho e de Recompensa sobre a Competência da Gestão de RH (Modelo 3).

Além de considerar a influência das variáveis independentes sobre a Competência da Gestão de RH os modelos da análise de regressão também foram avaliados em função de sua significância e multicolinearidade. O primeiro modelo foi testado para avaliar se as variáveis de controle poderiam influenciar significativamente a avaliação da Gestão de RH. Nos modelos seguintes essas variáveis foram mantidas para que as demais variáveis não fossem inflacionadas em função do não compartilhamento da variância explicada com outras variáveis independentes. O resultado do primeiro modelo mostrou que as variáveis de controle não convergem em um modelo ajustado e significativo para previsão da variação da atitude dos funcionários pesquisados em relação à Competência do RH, conforme esperado. Esse primeiro modelo apresentou valor não significativo $(F=2,071)$ e um pobre $R^{2}$ ajustado=0,01. Apesar de o modelo ser não significativo os índices de multicolinearidade (VIF e valores de tolerância) não indicaram alta correlação entre as variáveis independentes. 
Gestão estratégica de recursos humanos: quais práticas influenciam a avaliação dessa função pelos membros organizacionais?

\begin{tabular}{|c|c|c|c|c|c|c|}
\hline & \multicolumn{2}{|l|}{ Modelo 1} & \multicolumn{2}{|l|}{ Modelo 2} & \multicolumn{2}{|l|}{ Modelo 3} \\
\hline & $\beta$ & $T$ & $\beta$ & $t$ & $\beta$ & $t$ \\
\hline Constante & $5,436 * * *$ & $(40,266)$ & $0,730 * *$ & $(2,476)$ & 0,407 & $(1,336)$ \\
\hline Cargo (Nível $\mathbb{1}$ ) & $-0,741 * *$ & $(-2,375)$ & $-0,508 * *$ & $(-2,312)$ & $-0,444 * *$ & $(-2,052)$ \\
\hline Cargo (Nível 2) & $-0,130$ & $(-0,364)$ & $-0,105$ & $(-0,414)$ & $-0,152$ & $(-0,605)$ \\
\hline Tempo na empresa & 0,024 & $(1,010)$ & 0,026 & $(1,564)$ & 0,027 & $(1,630)$ \\
\hline Descrição de Cargos e Tarefas & & & $0,114 *$ & $(1,908)$ & $0,125 * *$ & $(2,118)$ \\
\hline Treinamento & & & $0,195^{* * *}$ & $(4,372)$ & $0,170 * * *$ & $(3,780)$ \\
\hline Ambiente Físico & & & 0,054 & $(0,987)$ & 0,052 & $(0,960)$ \\
\hline Autonomia & & & 0,040 & $(0,852)$ & 0,025 & $(0,532)$ \\
\hline Comunicação & & & $0,257 * * *$ & $(4,277)$ & $0,222 * * *$ & $(3,653)$ \\
\hline Avaliação de Desempenho & & & $0,142 * *$ & $(2,472)$ & $0,135 * *$ & $(2,377)$ \\
\hline Oportunidades de Carreira & & & 0,031 & $(0,774)$ & 0,004 & $(0,091)$ \\
\hline Remuneração & & & & & $0,131 * * *$ & $(2,698)$ \\
\hline Benefícios & & & & & 0,057 & $(1,184)$ \\
\hline$F$ & 2,071 & & $36,347^{* * *}$ & & $32,447^{* * *}$ & \\
\hline$R^{2}$ & 0,019 & & 0,538 & & 0,557 & \\
\hline$R^{2}$ Ajustado & 0,010 & & 0,523 & & 0,540 & \\
\hline$\Delta R^{2}$ Ajustado & & & 0,513 & & 0,017 & \\
\hline
\end{tabular}

Tabela 4: Regressão Hierárquica OLS (Ordinary Least Squares)

Fonte: dados primários.

$* * *(p<0,01)$

$* *(p<0,05)$

$*(p<0,10)$

O resultado do segundo modelo foi significativo a um $p$-value<0,01 $(F=36,347)$. O $\mathrm{R}^{2}$ ajustado $=0,523$ aponta que aproximadamente $52 \%$ da variação na avaliação da Competência da Gestão de Recursos Humanos é "explicada" em função das variáveis denominadas "Condições de Trabalho". Esse modelo indicou a Comunicação $(\beta=0,257, p$-value $<0,01)$, o Treinamento $(\beta=0,195$, $p$-value $<0,01)$, a Avaliação de Desempenho $(\beta=0,142, p$-value $<0,05)$ e a Descrição de Cargos e Tarefas $(\beta=0,114, p$-value $<0,10)$ como as principais variáveis de influência sobre a variável dependente. As estatísticas de multicolinearidade (VIF) e valores de tolerância estiveram de acordo com a referência recomendada: VIF entre 1,0 e 3,0 e valores de tolerância entre 0,10 e 1,0 (Hair Jr.et al., 1995). No terceiro modelo testado as variáveis de recompensa (remuneração e benefícios) foram agregadas às variáveis independentes do modelo anterior (Modelo 2). Esse modelo, assim como o anterior, se mostrou significativo $(F=36,347, p$-value $<0,01)$. Contudo, o $R^{2}$ ajustado=0,540 indicou não haver aumento substancial no poder preditivo do modelo 3 em comparação ao modelo 2, com a inclusão das variáveis de recompensa. As estatísticas de multicolinearidade desse modelo também estiveram de acordo com as referências recomendadas por Hair Jr.et al. (1995). Uma última análise de qualidade do modelo 3, considerado para teste das hipóteses foi a observação da distribuição do resíduos da variável dependente. Os dados de resíduos se mostraram normalmente distribuídos.

No terceiro modelo testado as variáveis de recompensa (remuneração e benefícios) foram agregadas às variáveis independentes do modelo anterior (Modelo 2). Esse modelo, assim como o anterior, se mostrou significativo $(F=36,347, p$-value $<0,01)$. Contudo, o $R^{2}$ ajustado $=0,540$ indicou não haver aumento substancial no poder preditivo do modelo $3 \mathrm{em}$ comparação ao modelo 2, com a inclusão das variáveis de recompensa. As estatísticas de multicolinearidade desse modelo também estiveram de acordo com as referências recomendadas por Hair Jr.et al. (1995). Uma última análise de qualidade do modelo 3, considerado para teste das hipóteses foi a observação da distribuição do resíduos da variável dependente. Os dados de resíduos se mostraram normalmente distribuídos. Assim como no modelo anterior, as variáveis Comunicação $(\beta=0,222, p$-value $<0,01)$, Treinamento $(\beta=0,170, p$-value $<0,01)$ e Avaliação de Desempenho $(\beta=0,135, p$-value $<0,05)$ foram as de maior poder de influência sobre a Competência da Gestão de Recursos Humanos. Além dessas variáveis as variáveis Remuneração $(\beta=0,131, p$-value $<0,01)$ e Descrição de Cargos e Tarefas $(\beta=0,125, p$ value $<0,05)$ também foram significativas. Isso equivale dizer: a Comunicação nas organizações é a responsabilidade da Gestão de Recursos Humanos que influencia mais fortemente a avaliação que os funcionários fazem da Competência da Gestão de RH de forma global. Já dentro das expectativas, as outras duas variáveis com maior influência sobre a Gestão de RH foram Treinamento e Avaliação de Desempenho.

Esse resultado coloca o aprimoramento da Comunicação, enquanto prática da Gestão de Recursos Humanos, como o caminho mais curto para desenvolvimento do status da área, ao menos aos olhos dos funcionários, como foi o caso da presente investigação. A força desse argumento na verdade não é nova, pois a comunicação está associada ao fluxo de informações entre organização e 
funcionário, bem como entre funcionários e entre departamentos, e sua centralidade quanto ao funcionamento das organizações é bem documentada na literatura. A Escola de Relações Humanas já destacou a comunicação informal dentro dos grupos como um dos principais condicionantes da produtividade. A Teoria dos Sistemas Abertos, bem como a Abordagem Sóciotécnica das organizações colocaram ênfase na interdependência informacional entre os vários elementos organizacionais. E os contingencialistas, como Lawrence e Lorsch (1967),destacaram que o processo necessário de dividir funções e especializar tarefas produz naturalmente uma fragmentação de relações entre os membros e departamentos organizacionais, que por sua vez exige esforços de integração. Esse fenômeno destacado por Lawrence e Lorsch (1967) foi denominado paradoxo diferenciação-integração. Ao mesmo tempo em que é necessário dividir tarefas e criar especialização e departamentos (diferenciação) existe a necessidade em integrar todos esses elementos.

De fato, o que há de novo trazido pela presente pesquisa, é a responsabilidade da área de RH pela comunicação nas organizações. Deve ficar claro que essa responsabilidade não se justifica apenas por uma necessidade estritamente instrumental, como a necessidade de fluxo de informações, mas, sobretudo na construção de uma realidade socialmente compartilhada, baseada em crenças e valores comuns. Isso significa a possibilidade de "construção" de uma identidade organizacional, bem como a construção da identidade profissional dos atores organizacionais. Logo, está sob a responsabilidade da gestão de $\mathrm{RH}$ o exercício de práticas de sensemaking e sensegiving (Luscher \&Lewis, 2008). Isso significa que, cabe à gestão de RH, fornecer e sustentar a arquitetura social necessária à construção de sentido (sensemaking) no trabalho por meio da entrega de sentido (sensegiving) por outros membros organizacionais. Tal exercício equivale a possibilitar ações simbólicas para disseminação das províncias de significados organizacionais, valores, que sincronizam a razão do "fazer" no trabalho com outras razões dos indivíduos e da organização na tarefa de ajuste ao seu ambiente externo. Esse trabalho de natureza interpretativa e simbólica sustenta a estabilidade e a conexão dos esquemas interpretativos dos atores sociais em dada organização. À luz desse processo de construção de consenso e da negociação entre visões de mundo diferentes, cabe à gestão de $\mathrm{RH}$, estabelecer seu status de função estratégica como imperativo para consecução dos objetivos organizacionais por meio de uma racionalidade coletiva e do comportamento cooperativo.

De modo geral, o que os resultados do presente estudo sugerem é a responsabilidade da Gestão de Recursos Humanos pelas práticas de atribuição e construção de sentido no trabalho dos membros organizacionais. Mas, se por um lado os resultados colocam essa tarefa à Gestão de RH, junto das práticas de treinamento, avaliação de desempenho e descrição de cargos como atribuições centrais da área, eles também revelam a Comunicação como principal exercício para reconstrução da sua legitimidade e status enquanto instituição. Os resultados apontam claramente um novo papel para a Gestão de RH, mais voltado para a interação empresa-funcionário, mais voltado para a forma e conteúdo do discurso, encarando o discurso como um recurso estratégico para as organizações, bem como para a própria área de conhecimento. Desse modo, esse parece ser o caminho para a renovação do papel e transformação efetiva dessa função na Gestão Estratégica de Recursos Humanos (Kochan, 2007, Kazmi \& Ahmad, 2001, Ferriset al., 1999, Jamrog \& Overholt, 2004).

\section{Conclusões}

Buscou-se com a realização desse trabalho avaliar o que a Gestão de Recursos Humanos precisa priorizar em suas ações para ser vista com "bons olhos" (como função organizacional competente) pelos funcionários. Essa linha de investigação vai ao encontro dos esforços de outros pesquisadores da área voltados à identificação das necessidades de renovação da Gestão de $\mathrm{RH}$ e crescimento de seu status enquanto disciplina e prática gerencial (e.g., Mahoney \& Deckop, 1986; Kochan, 2007, Kazmi \& Ahmad, 2001; Ferriset al., 1999). Após o levantamento com 347 funcionários, as escalas foram validadas e tiveram sua confiabilidade atestada pelos procedimentos supracitados. Testou-se então um modelo final de regressão múltipla hierárquica que assumiu como variáveis independentes as seguintes práticas ou sistemas de Gestão de Recursos Humanos: ( $X_{1=}$ treinamento; $X_{2=}$ descrição de cargos; $X_{3=}$ autonomia; $X_{4=}$ avaliação de desempenho; $X_{5=}$ oportunidades; $X_{6=}$ ambiente físico; $X_{7=}$ comunicação; $X_{8=}$ remuneração; $X_{9=}$ benefícios). A variável dependente foi: $\left(\gamma_{=}\right.$Competência da Gestão de $R H$ ). Os resultados permitiram tecer as seguintes conclusões:

- Entre todas as práticas de (i) condições de trabalho e (ii) recompensa, consideradas na pesquisa, as práticas de recompensa (Remuneração e Benefícios) apresentam menor poder explicativo sobre a variação na atitude dos funcionários em relação à Competência da Gestão de RH, o que coloca maior peso sobre as práticas denominadas Condições de Trabalho. 
- Entre as práticas de Condições de Trabalho as variáveis (i) Descrição de Cargos; (ii) Treinamento, (iii) Avaliação de Desempenho, e (iv) Comunicação são as principais práticas do sistema de RH que influenciam significativamente a Competência dessa área funcional.

- A prática de Comunicação é a que apresenta maior influência sobre o julgamento da Competência da Gestão de RH. Tal achado coloca em primeiro plano o desenvolvimento das ações de construção de sentido no trabalho para realização do papel estratégico da Gestão de Recursos Humanos.

- A Gestão de Recursos Humanos deveria assumir a Comunicação, via práticas de sensemaking e sensegiving (Luscher \&Lewis, 2008), como seu exercício nuclear, ou seja, colocar a Comunicação entre empresa-funcionário e entre departamentos como sua atribuição principal. Essa mudança de ênfase da Gestão de RH, de típicas atividades de treinamento para atividades de comunicação pode fazer valer efetivamente o papel de "parceiro estratégico" à área.

- Como última conclusão, a Gestão de Recursos Humanos deveria absorver entre suas ações principais, não apenas práticas instrumentais (e.g., recrutamento, seleção e treinamento), mas cada vez mais práticas de natureza simbólica (de significação).

Em resumo, é preciso ressaltar que além de identificar as principais práticas que exercem maior influência sobre a avaliação dos funcionários em relação à Gestão de RH, buscou-se também evidenciar a necessidade de avaliação do julgamento de diferentes atores sociais sobre essa função organizacional enquanto instituição social. Assim, espera-se que esse estudo seja visto apenas como o início de esforços voltados para a avaliação da Gestão de Recursos Humanos dentro e fora das organizações de trabalho. Nesse sentido, vale salientar a afirmação de Mamman e Rees (2004). De acordo com esses autores, apesar do crescimento no interesse pela teoria e prática da área de Gestão de Recursos Humanos (Human Resource Management), muito trabalho ainda permanece por ser feito em comparação à necessidade de reconhecimento desse campo. Por fim, sugere-se que futuros estudos tenham como alvo de estudo outras audiências da área de gestão de recursos humanos, além dos funcionários de organizações privadas, como executivos da área, presidentes de empresas e outros stakeholders. Outra sugestão é explorar as relações pesquisadas aqui em organizações públicas, de setores diferentes e com portes diferenciados.

\section{Referências}

Babbie, E. R. (1998). The practice of social research. California: Wadsworth Publishing Company. Babbie, E. R. (2001). Métodos de pesquisa de survey. Belo Horizonte: Editora UFMG.

Bohlander, G., Snell, S., \& Sherman, A. (2005). Administração de recursos humanos. São Paulo: Pioneira Thomson Learning.

Boudreau, J. W.,Ramstad, P. M. (2006). Talentship and HR measurement and analysis: from ROI to strategic organizational change. Human Resource Planning, 29(1), 25-33.

Boxall, P., Purcell, J., \& Wright, P. (Orgs.). (2007). The Oxford Handbook of human resource management. Oxford: University Press.

Camelo, C., Martín, F., Romero, P. M., \& Valle. R. (2004). Human resource management in Spain: is it possible to speak of a typical model? International Journal of Human Resource Management, 15(6), 935-958.

Clardy, A. (2008). The strategic role of human resource development in managing core competencies. Human Resource Development International, 11(5), 465-477.

Colbert, B. A., \& Kurucz, E. C. (2007). Three conceptions of triple bottom line business sustainability and the role for HRM. Human Resource Planning, 30(1), 21-29.

Collins, C. J., \& Clark, K. D. (2003). Strategic human resource practices, top management team social networks, and firm performance: the role of human resource practices in creating organizational competitive advantage. Academy of Management Journal, 46(6), 740-751.

Conway, E., \& Monks, K. (2008). HR practices and commitment to change: an employee-level analysis. Human Resource Management Journal, 18(1), 72-89. 
Denisi, A. S., Wilson, M. S., \& Biteman, J. (2014). Research and practice in HRM: a historical perspective. Resource Management Review, 24(1), 219-231.

Dutra, J. S. (2002). Gestão de pessoas. Editora Atlas.

Ferris, G, R., Hochwarter, W. A., Buckley, M. R., Harrell-Cook, G., \&Frink, D. D. (1999). Human resources management: some new directions. Journal of Management, 25(3), 385-415.

Fisher, A. L. (1998). A constituição do modelo competitivo de gestão de pessoas no Brasil: um estudo sobre as empresas consideradas exemplares. São Paulo: Departamento de Administração, Faculdade de Economia e Administração da Universidade de São Paulo. Tese de Doutorado.

Fleury, M. T. L. (2002). As pessoas na organização. São Paulo: Atlas.

Fornell, C., \& Larcker, D. F. (1981). Evaluating structural equation models with unobserved variables and measurement error. Journal of Marketing Research, 18(1), 39-50.

França. A. C. L. (2007). Práticas de recursos humanos. São Paulo: Atlas.

Graham, P. (1995). Mary Parker Follett: prophet of management. Boston: Harvard Business School Press.

Gvaramadze, I. (2008). Human resource development practice: the paradox of empowerment and individualization. Human Resource Development International, 11(2), 183-197.

Hair JR., J. F., Anderson, R. E., Tatham, R. L., \& Black, W. C. (1995). Multivariate data analysis. New Jersey: Prentice-Hall.

Innocenti, L., Pilati, M., \& Peluso, A. M. (2011). Trust as moderator in the relationship between HRM practices and employee attitudes. Human Resource Management Journal, 21(3), 303-317.

Jamrog, J. J., \& Overholt, M. H. (2004). Building a strategic HR function: continuing the evolution. Human Resource Planning, 27(2), 51-62.

Janssens, M., \& Steyaert, C. (2009). HRM and performance: a plea for reflexivity in HRM studies. Journal of Management Studies, 46(1), 143-155.

Katou, A. A., \& Budhwar, P. S. (2006). Human resource management systems and organizational performance: a test of a mediating model in the Greek manufacturing context. International journal of Human Resource Management, 17(7), 1223-1253.

Kazmi, A., \& Ahmad, F. (2001). Differing approaches to strategic management. Journal of Management Research, 1(3), 133-140.

Kochan, T. A. (2007). Social legitimacy of the HRM profession: a US perspective. In: Boxall, P.; Purcell, J., \& Wright, P. (Orgs.). The Oxford Handbook of human resource management. Oxford: University Press, p. 599-620.

Lawler III, E. E., \& Mohrman, S. A. (2003). HR as a strategic partner: what does it take to make it happen? Human Resource Planning, 26(5), 15-29.

Lawler III, E. E., Levenson, A., \& Boudreau, J. W. (2004). HR metrics and analytics: use and impact. Human Resource Planning, 27(4), 27-35.

Lawrence, P. R., \& Lorsch, J. (1967). Organization and environment. Boston: Harvard Business School Press.

Lawson, T. E., \& Hepp, R. L. (2001). Measuring the performance impact of human resource initiatives. Human Resource Planning, 24(4), 36-44.

Lounsbury, J. W., Steel, R. P.,Gibson, L. W., \& Drost, A. W. (2008). Personality traits and career satisfaction of human resource professionals. Human Resource Development International, $11(4)$, $351-366$.

Luscher, L. S., \& Lewis, M. W. (2008). Organizational change and managerial sensemaking: working through paradox. Academy of Management journal, 51(2), 221-240.

Mahoney, T. A., \& Deckop, J. R. (1986). Evolution of concept and practice in personnel administration/human resource management (PA/HRM). journal of Management, 12(2), 223-241.

Mamman, A., \& Rees, C. J. (2004). How retail is the rhetoric? A survey of American HR managers' view about HRM. International Journal of Management, 21(1), 115-129. 
Mathis, R. L., \& Jackson, J. H. (2008). Human resource management. Mason: Thomson Learning. Neuman, L. W. (1997). Social research methods: qualitative and quantitative approaches. Boston: Allyn \& Bacon.

Paauwe, J. (2009). HRM and performance: achievements, methodological issues and prospects. Journal of Management Studies, 46(1), 129-142.

Parker, L. D. (1984). Control in organizational life: the contribution of Mary Parker Follett. Academy of Management Review, 9(4), 736-745.

Premeaux, S. F., \& Breaux, D. (2007). Crisis management of human resources: lessons from hurricanes Katrina and Rita. Human Resource Planning, 30(3), 39-47.

Ramlall, S. J. (2003). Measuring human resource management's effectiveness in improving performance. Human Resource Planning, 26(4), 51-62.

Rau, B. (2012). The diffusion of HR practices in unions. Human Resource Management journal, 22(1), 27-42.

Renckly, R. G. (2004). Human resources. NY: Barron's Business Library.

Rodríguez, J. M., \& Ventura, J. (2003). Human resource management systems and organizational performance: an analysis of the Spanish manufacturing industry. International journal of Human Resource Management, 14(7), 1206-1226.

Schumacker, R. E., \& Lomax, R. G. (2004). A beginner's guide to structural equation modeling. Mahwah: Lawrence Erlbaum Associates Publishers.

Stone, D. L. (2014). Human resource management: past, present and future. Human Resource Management Review, 24(1), 193-195.

Thompson, P. (2011). The trouble with the HRM. Human Resource Management Journal, 21(4), 355367.

Vickers, M. R. (2005). Business ethics and the HR role: past, present, and future. Human Resource Planning, 28(1), 26-32.

Visser, M. (2010). Configurations of human resource practices and battlefield performance: a comparison of two armies. Human Resource Management Journal, 20(4), 340-349.

Vosburg, R. M. (2007). The evolution of HR: developing HR as an internal consulting organization. Human Resource Planning, 30(3), 11-23.

Wood Jr., T., Tonelli, M. J., \& Cooke, B. (2012). Para onde vai a gestão de pessoas. GV-executivo, $11(2), 20-24$.

Wright, P. M., \& McMahan, G. C. (1992). Theoretical perspectives for strategic human resource management. Journal of Management, 18(2), 295-320.

Zientara, P. (2009). Employment of older workers in Polish SMEs: employer attitudes and perceptions, employee motivations and expectations. Human Resource Development International, 12(2), 135-153. 